\title{
Efecto de los componentes del síndrome metabólico en la función pulmonar. El inesperado papel del colesterol ligado a lipoproteínas de alta densidad
}

\author{
Effect of the components of the metabolic syndrome on pulmonary function. \\ The unexpected role of high-density lipoprotein cholesterol
}

\section{Saúl Huerta-Ramírez ${ }^{1 *}$, Angélica Paniagua-Pérez², David Castro-Serna ${ }^{1}$, Andrés Ledesma-Velázquez¹, Alberto Rubio-Guerra ${ }^{3}$ y Germán Vargas-Ayala ${ }^{1}$}

${ }^{1}$ Servicio de Medicina Interna, SEDESA, Hospital General Ticomán; ${ }^{2}$ Servicio de Medicina Interna, Hospital General de Zona 58, Instituto Mexicano del Seguro Social; ' 3 Jefatura de Enseñanza e Investigación, SEDESA, Hospital General Ticomán. Ciudad de México, México

\begin{abstract}
Resumen
Antecedentes: El síndrome metabólico es un estado que predispone a enfermedad cardiovascular y diabetes mellitus. Además, puede repercutir en la función hepática, en patologías neoplásicas y en la función pulmonar. Nuestro objetivo es analizar el efecto del síndrome metabólico y sus componentes sobre la función pulmonar. Método: Se evaluaron 110 sujetos de la Ciudad de México a quienes se realizaron mediciones antropométricas, determinación de glucosa, triglicéridos y colesterol ligado a lipoproteínas de alta densidad (HDL). Se les practicó una espirometría simple. Se realizó el diagnóstico de síndrome metabólico siguiendo los criterios NCEP-ATPIII. Resultados: De 110 individuos, 90 (82\%) fueron mujeres y 20 hombres (18\%), y $71(65 \%)$ presentaron síndrome metabólico. Los sujetos con obesidad central tuvieron una capacidad vital forzada (CVF) menor que aquellos sin obesidad central (2.72 vs. $3.11 \mathrm{l} ; p<0.05$ ). Los que presentaron colesterol HDL bajo tuvieron mejores resultados espirométricos que los sujetos con colesterol HDL normal (volumen espiratorio forzado en el primer segundo [VEF1] 2.36 vs. 1.85 l; $p<0.05$ ), mejor CVF (2.95 vs. 2.45 l; $p<0.05$ ) y mejor relación VEF1/CVF (78 vs. 74; $p<0.05)$. Los sujetos hipertensos presentaron menores volúmenes en VEF1 (1.91 vs. 2.38; $p<0.05)$ y CVF (2.49 vs. 2.99; $p<0.05)$. Conclusión: No existe diferencia en los volúmenes espirométricos de pacientes con síndrome metabólico al compararlos con sujetos metabólicamente sanos. Solo la obesidad central y la hipertensión arterial se asocian con disminución del VEF1 y la CVF. Un hallazgo inesperado es la correlación negativa entre los valores de colesterol HDL y la función pulmonar.
\end{abstract}

PALABRAS CLAVE: Espirometría. Síndrome metabólico. Pulmón. Colesterol HDL.

\begin{abstract}
Background: Metabolic syndrome is a condition that predisposes to cardiovascular disease and diabetes mellitus. In addition, it can have effects over neoplastic pathologies, liver and pulmonary function. Our objective is to analyze the effect of the metabolic syndrome and its components on pulmonary function. Method: 110 subjects from Mexico City were evaluated and anthropometric measurements, glucose determination, triglycerides and high-density lipoprotein (HDL) cholesterol were made. They underwent a simple spirometry. Diagnosis of metabolic syndrome was made following the NCEP-ATPIII criteria. Results: Of 110 individuals, 90 (82\%) were women and 20 men (18\%); 71 subjects (65\%) presented metabolic syndrome. Subjects with central obesity had a forced vital capacity (FVC) lower than subjects without central obesity (2.72 vs. 3.11 liters; $p<0.05)$.
\end{abstract}

\author{
Correspondencia: \\ *Saúl Huerta Ramírez \\ Plan de San Luis, s/n \\ Col. Ticomán, Del. Gustavo A. Madero \\ C.P. 07320, Ciudad de México, México \\ E-mail: saul.huerta@ hotmail.com
}

Fecha de recepción: 08-12-2017

Fecha de aceptación: 11-04-2018

DOI: 10.24875/CIRU.M18000030
Cir Cir. 2018;86:175-181

Contents available at PubMed www.cirugiaycirujanos.com 
Those with low HDL had better spirometric results than subjects with normal HDL (FEV1 2.36 vs. 1.85 liters; $p<0.05$ ), FVC (2.95 vs. 2.45 liters; $p<0.05)$ and FEV1/FVC ratio $(0.78$ vs.74; $p<0.05)$. Hypertensive subjects presented lower volumes in FEV1 (1.91 vs. 2.38; $p<0.05)$ and FVC (2.49 vs. 2.99; $p<0.05)$. Conclusion: There is no difference between the spirometry volumes of patients with metabolic syndrome versus the metabolically healthy subjects. The only factors associated with a decrease in FEV1 and FVC are central obesity and arterial hypertension. An unexpected finding was the negative correlation between HDL levels and lung function.

KEY WORDS: Spirometry. Metabolic syndrome. Lung. HDL cholesterol.

\section{Antecedentes}

El síndrome metabólico (SMet) se define como un grupo de características clínicas que incluyen obesidad abdominal, hiperglucemia, triglicéridos elevados, hipertensión arterial y bajos valores de colesterol ligado a lipoproteínas de alta densidad (HDL)1. Este síndrome es altamente frecuente en la población mundial, y su prevalencia utilizando los criterios diagnósticos del NCEP-ATPIII se ha reportado hasta en el $22 \%$ de la población de los países desarrollados y el $26 \%$ en población mexicana ${ }^{2,3}$.

El SMet constituye un importante factor de riesgo para enfermedad cardiovascular (infarto de miocardio, enfermedad vascular cerebral, insuficiencia arterial), así como para el desarrollo de diabetes mellitus tipo 2. Recientemente se ha reportado que el SMet puede influir en el desarrollo de esteatosis hepática no alcohólica e incluso en diversos tipos de cáncer4. En la actualidad se está conjuntando evidencia del impacto que el SMet podría tener en diferentes aspectos de la función pulmonar ${ }^{5}$.

Existen algunos estudios en población asiática y estadounidense que indican que el SMet tiene cierta asociación con un patrón pulmonar restrictivo; sin embargo, aún hay controversia en la contribución relativa que cada componente del síndrome tiene en la afección pulmonar ${ }^{6}$. Se ha sugerido que cada componente del síndrome metabólico puede tener una influencia diferente en la fisiopatología respiratoria según el grupo racial y el sexo ${ }^{7}$.

Dado que la relación entre el SMet y la función pulmonar ha sido poco estudiada en población latina, y el entendimiento de que esta posible asociación puede tener implicaciones importantes en el manejo de estos pacientes ${ }^{8}$, el propósito de este estudio es analizar el efecto que tiene el SMet y cada uno de sus componentes sobre los volúmenes pulmonares medidos por espirometría en habitantes de la Ciudad de México.

\section{Método}

Se realizó un estudio transversal analítico con muestreo por conveniencia, para lo cual se invitó a participar a sujetos elegibles, que fueron atendidos en diferentes servicios de consulta externa del Hospital General de Ticomán en la Ciudad de México durante el primer semestre del año 2017. Se incluyeron individuos de 18 a 75 años que no tuvieran enfermedad pulmonar conocida ni exposiciones de riesgo para patología pulmonar, que aceptaran responder un cuestionario y otorgar una muestra de sangre, y que tuvieran la capacidad para realizar las maniobras que requiere el estudio de espirometría.

A los sujetos que aceptaron participar se les realizó un cuestionario para registrar variables demográficas como sexo, edad, patologías conocidas y exposiciones de riesgo pulmonar. Se les realizaron mediciones antropométricas (peso, talla, índice de masa corporal y circunferencia abdominal) y registro de presión arterial, siguiendo los lineamientos establecidos por la Organización Mundial de la Salud. Se les tomó muestra de sangre en ayuno para la determinación de glucosa, triglicéridos y colesterol HDL. La glucosa se midió por el método de glucosa oxidasa con un equipo Beckman Coulder DxC600; los triglicéridos y el colesterol HDL se midieron con el analizador automatizado DxC600.

A partir de los resultados del cuestionario, las mediciones antropométricas y los estudios de laboratorio, los sujetos fueron diagnosticados con o sin SMet. En apego a los criterios armonizados publicados en 2009 por el Joint Scientific Statemet ${ }^{9}$, se diagnosticó SMet cuando el sujeto cumplió al menos tres de los siguientes: glucosa > $100 \mathrm{mg} / \mathrm{dl}$ o estar en tratamiento para diabetes tipo 2; perímetro abdominal $>90 \mathrm{~cm}$ en hombres y $>80 \mathrm{~cm}$ en mujeres; triglicéridos $>150 \mathrm{mg} / \mathrm{dl}$ o estar en tratamiento para disminuirlos; colesterol $\mathrm{HDL}<40 \mathrm{mg} / \mathrm{dl}$ en hombres y $<50 \mathrm{mg} / \mathrm{dl}$ en mujeres; y presión arterial $>140 / 90 \mathrm{~mm} / \mathrm{Hg} 0$ estar en tratamiento antihipertensivo. 
Finalmente, se les practicó un estudio de espirometría simple con el espirómetro marca Medizintechnik, TrueFlow Easy on PC. Se excluyeron de la espirometría los sujetos con dolor torácico; cirugía ocular, abdominal u ortopédica reciente; que utilizaran oxígeno suplementario; o con incapacidad para realizar esfuerzo inspiratorio o espiratorio máximo. El estudio se realizó por parte de personal médico capacitado. Se requirieron tres esfuerzos aceptables y solo se incluyeron los resultados de calidad $A, B$ y $C$ según los lineamientos de la American Thoracic Society, y la interpretación fue con apego a los valores predichos por NAHNES III.

El análisis estadístico de los datos se realizó utilizando el programa STATA 11.0. Se reportó la estadística descriptiva mediante frecuencias y porcentajes para variable nominales, así como medias o medianas (según el caso) para variables cuantitativas. Para la estadística inferencial se compararon las medias de los parámetros pulmonares de interés entre los grupos de sujetos de acuerdo con sus aspectos metabólicos. Se realizaron correlaciones simples entre algunas variables metabólicas y espirométricas.

\section{Resultados}

Se incluyeron en el estudio 110 individuos, de los que 90 (82\%) fueron mujeres y 20 (18\%) hombres. Del total, 71 sujetos $(65 \%)$ cumplieron criterios para SMet: $61(55 \%)$ resultaron con el factor de hiperglucemia, $91(83 \%)$ tenían el perímetro abdominal aumentado, 67 (62\%) tuvieron triglicéridos elevados, 74 (67\%) presentaron colesterol HDL bajo y $44(40 \%)$ tenían hipertensión arterial (Tabla 1).

Al comparar los volúmenes espirométricos de los individuos con y sin SMet no encontramos diferencia estadísticamente significativa (Tabla 2).

En cuanto al análisis de cada factor del SMet y su repercusión en los volúmenes pulmonares, se encontró que los sujetos con circunferencia abdominal aumentada presentaron menor capacidad vital forzada (CVF) (2.72 vs. $3.11 \mathrm{l} ; \mathrm{p}=0.04$ ) que los sujetos con circunferencia abdominal normal. Los individuos con hipertensión arterial presentaron menor volumen espiratorio forzado en el primer segundo (VEF1) (1.91 vs. 2.38 I; $p=0.01$ ) y menor CVF (2.49 vs. $2.99 ; p=0.01$ ) que los no hipertensos. Los factores hipertrigliceridemia e hiperglucemia no se asociaron con alteraciones en la espirometría. Los individuos con colesterol HDL normal o aumentado presentaron menores volúmenes de VEF1 (2.36 vs. $1.85 \mathrm{l} ; \mathrm{p}=0.01$ ), CVF (2.95 vs. 2.45 ,
Tabla 1. Características clínicas de la muestra

\begin{tabular}{|c|c|c|}
\hline Característica (N = 110) & Frecuencia & $(\%)$ \\
\hline \multicolumn{3}{|l|}{ Sexo } \\
\hline Hombres & 20 & $(18)$ \\
\hline Mujeres & 90 & (82) \\
\hline \multicolumn{3}{|l|}{ Síndrome metabólico } \\
\hline Con & 71 & (65) \\
\hline $\operatorname{Sin}$ & 39 & (35) \\
\hline \multicolumn{3}{|l|}{ Hiperglucemia } \\
\hline Con & 61 & (55) \\
\hline $\operatorname{Sin}$ & 49 & $(55)$ \\
\hline \multicolumn{3}{|l|}{ Perímetro abdominal } \\
\hline Normal & 19 & (17) \\
\hline Aumentado & 91 & (83) \\
\hline \multicolumn{3}{|l|}{ Triglicéridos } \\
\hline Normal & 41 & (38) \\
\hline$>150 \mathrm{mg}$ & 67 & (62) \\
\hline \multicolumn{3}{|l|}{ Colesterol HDL } \\
\hline Normal & 36 & (33) \\
\hline Bajo & 74 & (67) \\
\hline \multicolumn{3}{|l|}{ Hipertensión arterial } \\
\hline $\operatorname{Sin}$ & 66 & $(60)$ \\
\hline Con & 44 & $(40)$ \\
\hline \multicolumn{3}{|l|}{ Cuantitativas $(\mathrm{N}=110)$} \\
\hline & Mediana & p25-75 \\
\hline Edad (años)* & 54 & \pm 13 \\
\hline IMC & 27.5 & 25.3-31.4 \\
\hline $\mathrm{PA}(\mathrm{cm})$ & 95 & $88-100$ \\
\hline Glucosa (mg/dl) & 98.5 & $91-111$ \\
\hline Triglicéridos (mg/dl) & 173 & $129-238$ \\
\hline Colesterol HDL (mg/dl) & 43 & $35-51$ \\
\hline
\end{tabular}

*Variable con distribución paramétrica, se reportan media y desviación estándar. Todas las variables siguieron una distribución no paramétrica, por lo que se reportan medianas y percentiles 25-75.

HDL: lipoproteínas de alta densidad; IMC: índice de masa corporal; PA: perímetro abdominal

$p=0.01)$ y relación VEF1/VCF (0.78 vs. $0.74, p=0.01)$ que los sujetos con HDL bajo (Tabla 3).

Para establecer el grado de asociación que el colesterol HDL presenta con la disminución de los volúmenes pulmonares se realizó una regresión lineal simple, en la que se observa una correlación inversa significativa entre el colesterol HDL y los tres parámetros pulmonares (Tabla 4 y Figs. 1 a 3).

\section{Discusión}

Nuestros resultados indican que en una muestra de población mexicana no existe diferencia entre los volúmenes pulmonares de los pacientes que cumplieron 
Tabla 2. Volúmenes espirométricos en pacientes con y sin síndrome metabólico

\begin{tabular}{lcrr}
\hline Función pulmonar por espirometría & Sin síndrome metabólico $(\mathbf{n = 3 9 )}$ & Con síndrome metabólico $(\mathbf{n = 7 1 )}$ & $\mathbf{p}$ \\
\hline VEF1 (I) & 2.16 & 2.21 & 0.71 \\
\%VEF1 & 88.3 & 87.9 & 0.90 \\
CVF $($ I) & 2.79 & 2.79 & 0.9 \\
\%CVF & 89.6 & 87.6 & 0.47 \\
VEF1/CVF & 75 & 78 & 0.10 \\
\hline
\end{tabular}

CVF: capacidad vital forzada; VEF1: volumen espiratorio forzado en el primer segundo.

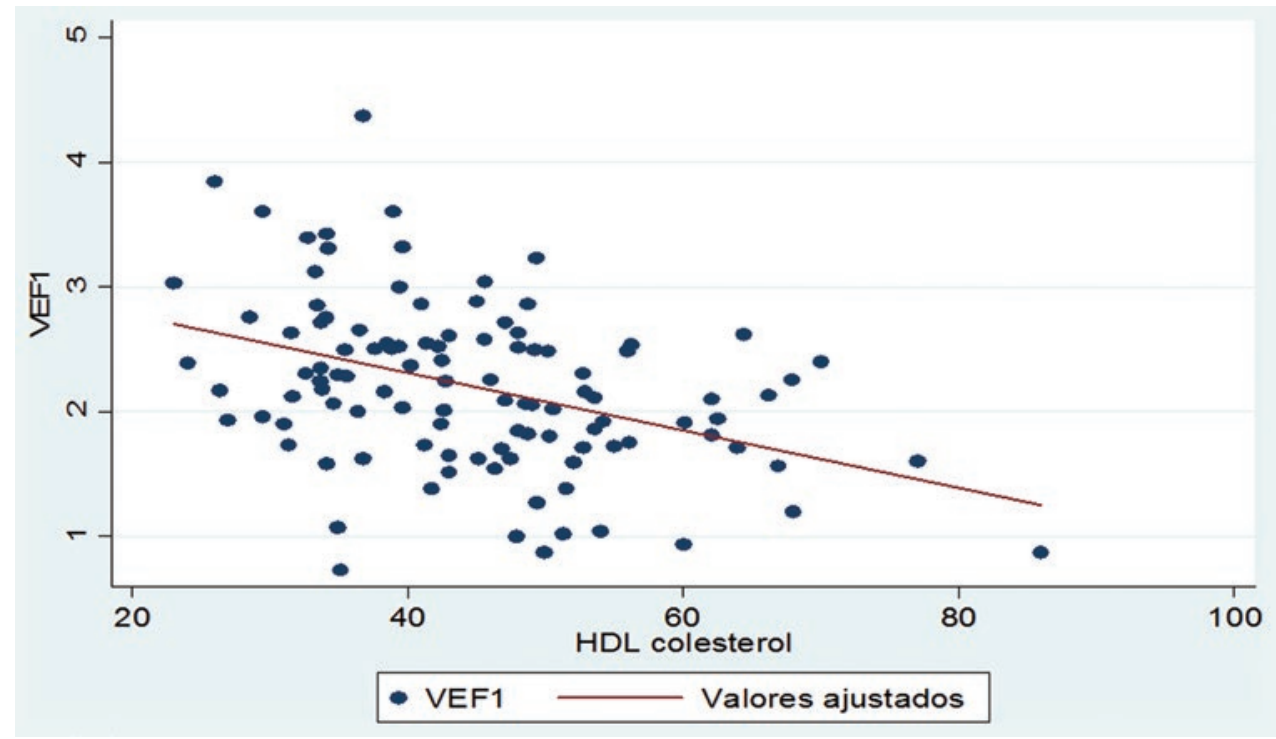

Figura 1. Regresión lineal simple entre el VEF1 (l) y el colesterol HDL (mg/dl).

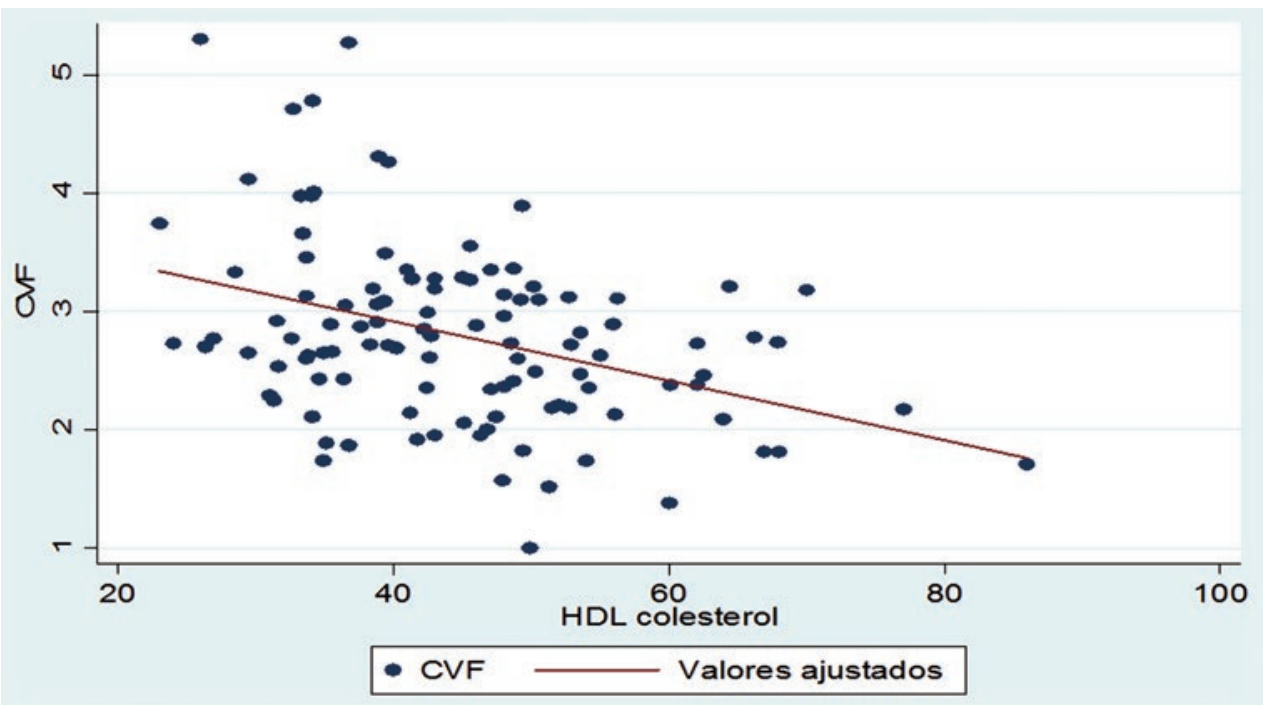

Figura 2. Regresión lineal simple entre la CVF (l) y el colesterol HDL (mg/dl).

criterios de SMet (según los criterios armonizados de 2009) y los de aquellos pacientes sin el síndrome. Sin embargo, cuando se analiza por separado la repercusión que cada componente del síndrome tiene sobre la función pulmonar, observamos que los sujetos con el factor circunferencia abdominal elevada (obesidad central) presentan menor CVF que aquellos sin este tipo de obesidad, y que los sujetos con el factor 


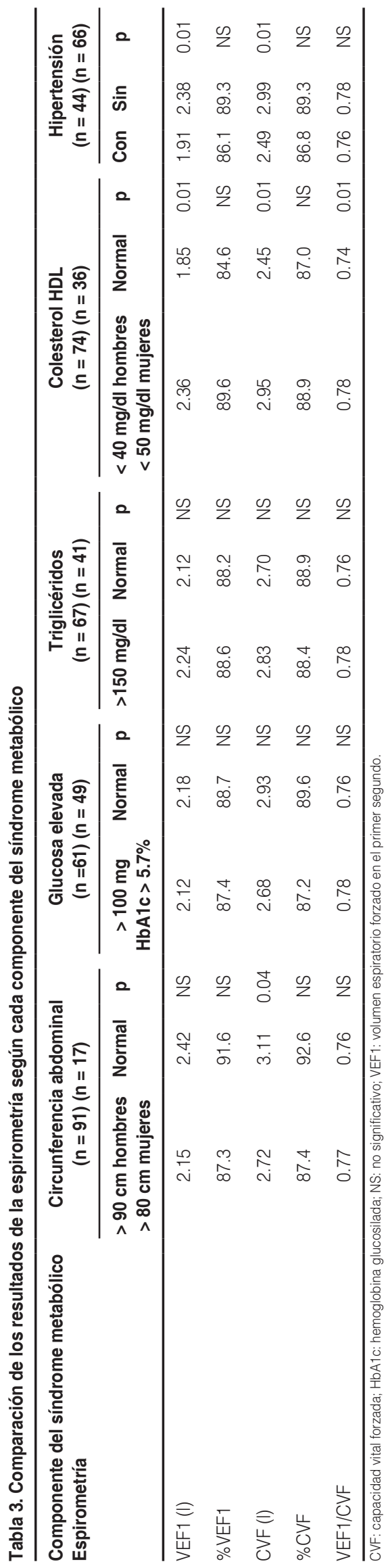

Tabla 4. Correlación (mediante regresión lineal simple) entre la función pulmonar y el colesterol HDL

\begin{tabular}{llccc}
\hline Colesterol HDL & Espirometría & $\boldsymbol{\beta}$ & $\mathbf{R}$ & $\mathbf{p}$ \\
\hline & VEF1 & -0.02 & -0.04 & 0.001 \\
& CVF & -0.02 & -0.38 & 0.001 \\
& VEF1/CVF & -0.001 & -0.22 & 0.01 \\
\hline B: coeficiente de correlación beta; CVF: capacidad vital forzada; R: correlación; & \\
VEF1: volumen espiratorio forzado en el primer segundo. & &
\end{tabular}

hipertensión arterial presentan menor VEF1 y CVF que los individuos no hipertensos. De manera contraria, los sujetos con el componente colesterol HDL bajo presentaron mayores volúmenes espirométricos que los sujetos con el colesterol HDL normal o alto.

Estudios previos con diseño de casos y controles ${ }^{10}$ identificaron inicialmente a los pacientes con patrones pulmonares obstructivos y restrictivos para analizar la prevalencia del SMet entre ellos, y señalaron que ambos patrones se asociaron con mayor presencia de SMet. Marquis, et al. ${ }^{11}$ reportaron en 38 pacientes con EPOC y 34 controles una prevalencia de SMet del 47 y el $21 \%$, respectivamente, mientras que un estudio en Taiwán ${ }^{12}$ que analizó más de 40,000 sujetos mostró que el patrón pulmonar restrictivo, pero no el obstructivo, se asoció con la presencia de SMet (odds ratio: 1.22). El diseño de estos abordajes difiere del nuestro, ya que ellos partieron del diagnóstico de alteración funcional pulmonar restrictiva u obstructiva para observar la prevalencia del SMet, mientras que nosotros identificamos primero el SMet para buscar luego el comportamiento de los volúmenes espirométricos entre los sujetos con el síndrome y sin él.

Un abordaje similar al nuestro, reportando los parámetros pulmonares en función de la presencia de SMet y cada uno de sus componentes, lo encontramos en tres reportes. El primero de ellos ${ }^{13}$, con datos del Strong Heart Study que explora población de indígenas norteamericanos, encontró que los valores medios de FEV1, FEV1\%, CVF y CVF\% fueron menores en los sujetos con SMet. Este estudio también analizó por separado dos componentes del SMet y su efecto en la función pulmonar, y se halló que tanto la diabetes mellitus como la obesidad central se asociaron con una disminución de los parámetros pulmonares. Un análisis en población norteamericana derivado de la cohorte NANHES ${ }^{8}$ reportó que en los sujetos con SMet es ligeramente más prevalente un patrón restrictivo de la función pulmonar, y que cada uno de los cinco componentes por separado también se asocia con una restricción pulmonar leve. Una investigación 


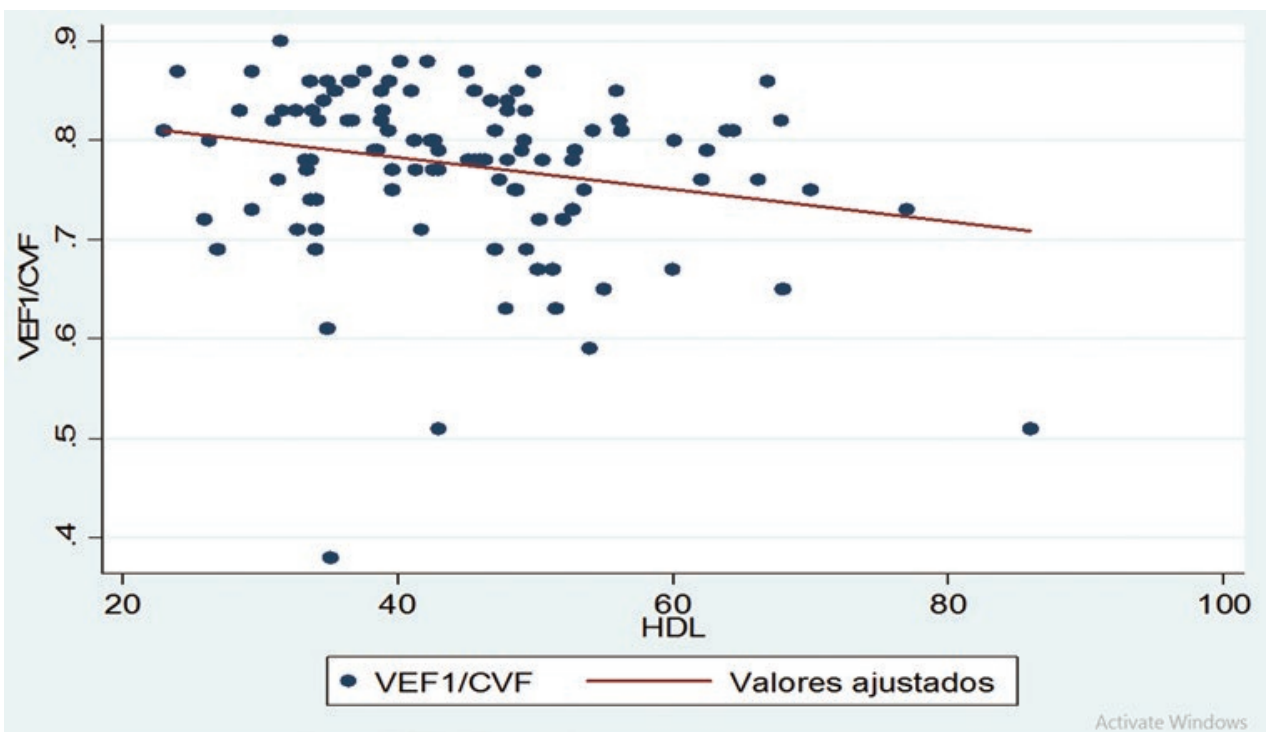

Figura 3. Regresión lineal simple entre el índice VEF1/CVF (\%) y el colesterol HDL (mg/dl).

realizada en Corea ${ }^{14}$ sugirió una posible influencia del sexo en el efecto que el SMet tiene sobre el pulmón. Estudiaron 1370 sujetos con SMet y observaron que solo los hombres presentaron una ligera disminución en el FEV1 y la CVF. Este último estudio halló que no todos los factores del SMet repercuten de manera negativa en la espirometría; de hecho, el colesterol HDL y la circunferencia abdominal se asocian con mejores volúmenes pulmonares. Este último hallazgo es concordante con nuestros resultados.

Como se puede observar, en estos reportes hay ciertas concordancias, pero también discrepancias en cuanto al efecto que los diferentes componentes del SMet tienen en la función pulmonar. Esta situación podría ser explicada no solo por la distinta metodología empleada y el tipo de sujetos incluidos en cada investigación, sino también por diferencias genéticas y raciales que condicionan diferentes comportamientos de los componentes del SMet, sobre todo las lipoproteínas ${ }^{15}$.

En nuestro estudio observamos que los sujetos con valores normales o elevados de colesterol HDL tienen menores volúmenes pulmonares que los sujetos con HDL bajo. Este es un hallazgo que calificamos como «inesperado», ya que debido a las funciones antiaterogénicas, antiinflamatorias, antioxidantes e incluso antiapoptóticas atribuidas a esta lipoproteína se especularía que sus valores elevados tendrían un factor benéfico para la función pulmonar ${ }^{16-18}$. Si bien es cierto que al colesterol HDL se le han atribuido las funciones citadas, recientemente está cambiando el paradigma del colesterol HDL como protector para la aterogénesis, y un ejemplo es la demostración de que la función de eflujo de colesterol, que inicialmente se atribuyó a esta lipoproteína, es independiente de ella. De hecho, se ha reconocido que su capacidad antiinflamatoria y movilizadora del colesterol se ve seriamente afectada con la oxidación de las proteínas asociadas a HDL, y que incluso este HDL disfuncional puede ser proinflamatorio ${ }^{19}$. Este nuevo enfoque sobre los aspectos fisiológicos del colesterol HDL podría explicar en parte nuestro hallazgo de que los sujetos con valores elevados presenten menores volúmenes pulmonares.

Coincidiendo con nosotros, un estudio genético y molecular, realizado en dos razas (afroamericanos y europeo-americanos), ha reportado dos polimorfismos en genes asociados con la apolipoproteína $M$ (APOM) que se relacionan con una disminución de la función pulmonar. La APOM es una lipoproteína que acompaña al colesterol HDL, y un cambio en su expresión genética altera la calidad y la función del colesterol HDL. De hecho, este mismo estudio ha encontrado que valores altos de HDL colesterol se asocian con una relación VEF1/CVF disminuida ${ }^{15}$.

El actual estudio tiene diversas limitantes. Al ser un estudio transversal no se puede establecer relación causal entre los factores metabólicos y las alteraciones pulmonares. La población en la que se realizó podría no ser representativa de la población mexicana general, ya que se hizo en sujetos que eran población cautiva de un hospital. El tamaño de la muestra es limitado y las conclusiones no deben ser generalizables. Se requieren más estudios, y en diferentes poblaciones, 
que exploren el efecto que los factores del SMet tienen sobre la función pulmonar, dado que hay indicios de que esta asociación es variable en cada raza.

\section{Conclusiones}

En población mexicana, los sujetos con SMet presentan una función pulmonar similar a los sujetos sin SMet. Sin embargo, cada componente del SMet desempeña un papel diferente en la función pulmonar, ya que los sujetos con circunferencia abdominal aumentada, con hipertensión arterial sistémica y con colesterol HDL normal o alto presentan menores volúmenes por espirometría que los sujetos sin estos factores.

\section{Bibliografía}

1. Eckel RH, Grundy SM, Zimmet PZ. The metabolic syndrome. Lancet. 2005;365:1415-28.

2. Beltrán $\mathrm{H}$, Harhay MO, Harhay MM, McElligott S. Prevalence and trends of metabolic syndrome in the adult U.S. population, 1999-2010. J Am Coll Cardiol. 2013;62:697-703.

3. Wacher NH. Epidemiología del síndrome metabólico. Gac Med Mex 2009:145:384-91.

4. Brede S, Serfling G, Klement J, Schmid SM, Lehnert H. Clinical scenario of the metabolic syndrome. Visc Med. 2016;32:336-41.

5. Leone N, Courbon D, Thomas F, Bean K, Jégo B, Leynaert B, et al. Lung function impairment and metabolic syndrome: the critical role of abdominal obesity. Am J Respir Crit Care Med. 2009;179:509-16.

6. Baffi CW, Wood L, Winnica D, Strollo PJ, Gladwin MT, Que LG, et al. Metabolic syndrome and the lung. Chest. 2016;149:1525-34.

7. Chen WL, Wang CC, Wu LW, Kao TW, Chan JY, Chen YJ, et al. Relationship between lung function and metabolic syndrome. PLoS One. 2014;9:e108989.
8. Ford ES, Cunningham TJ, Mercado Cl. Lung function and metabolic syndrome: findings of National Health and Nutrition Examination Survey 2007-2010. J Diabetes. 2014;6:603-13.

9. Alberti KG, Eckel RH, Grundy SM, Zimmet PZ, Cleeman JI, Donato KA, et al. Harmonizing the metabolic syndrome: a joint interim statement of the International Diabetes Federation Task Force on Epidemiology and Prevention; National Heart, Lung, and Blood Institute; American Heart Association; World Heart Federation; International Atherosclerosis Society; and International Association for the Study of Obesity. Circulation. 2009;120:1640-5.

10. Reed RM, lacono A, DeFilippis A, Eberlein M, Girgis RE, Jones S. Advanced chronic obstructive pulmonary disease is associated with high levels of high-density lipoprotein cholesterol. J Heart Lung Transplant. 2011;30:674-8.

11. Marquis K, Maltais F, Duguay V, Bezeau AM, LeBlanc P, Jobin J, et al. The metabolic syndrome in patients with chronic obstructive pulmonary disease. J Cardiopulm Rehabil. 2005;25:226-32.

12. Lin WY, Yao CA, Wang HC, Huang KC. Impaired lung function is associated with obesity and metabolic syndrome in adults. Obesity (Silver Spring). 2006;14:1654-61.

13. Yeh F, Dixon AE, Marion S, Shaefer C, Zhang Y, Best LG, et al. Obesity in adults is associated with reduced lung function in metabolic syndrome and diabetes: the Strong Heart Study. Diabetes Care. 2011; 34:2306-13.

14. Bae MS, Han JH, Kim JH, Kim YJ, Lee KJ, Kwon KY. The relationship between metabolic syndrome and pulmonary function. Korean J Fam Med. 2012;33:70-8.

15. Burkart KM, Manichaikul A, Wilk JB, Ahmed FS, Burke GL, Enright P, et al. APOM and high-density lipoprotein cholesterol are associated with lung function and per cent emphysema. Eur Respir J. 2014; 43:1003-17.

16. Gordon S, Durairaj A, Lu JL, Davidson WS. High-density lipoprotein proteomics: identifying new drug targets and biomarkers by understanding functionality. Curr Cardiovasc Risk Rep. 2010;4:1-8.

17. Pian MS, Dobbs LG. Lipoprotein-stimulated surfactant secretion in alveolar type II cells: mediation by heterotrimeric G proteins. Am J Physiol. 1997;273:634-9.

18. Kolleck I, Schlame M, Fechner H, Looman AC, Wissel H, Rüstow B. HDL is the major source of vitamin $E$ for type II pneumocytes. Free Radic Biol Med. 1999;27:882-90.

19. Khera AV, Cuchel M, de la Llera-Moya M, Rodrigues A, Burke MF, Jafri K, et al. Cholesterol efflux capacity, high-density lipoprotein function, and atherosclerosis. N Engl J Med. 2011;364:127-35. 